\title{
Interventions to Increase Depression Treatment Initiation in Primary Care Patients: a Systematic Review
}

\author{
Nathalie Moise, MD, MS ${ }^{7}$, Louise Falzon, PGDiplnf ${ }^{7}$, Megan Obi, BA ${ }^{2}$, Siqin Ye, MD, $M S^{7}$, \\ Sapana Patel, $\mathrm{PhD}^{3,4}$, Christopher Gonzalez, MD', Kelsey Bryant, MD, MPH${ }^{5}$, and lan M. Kronish, \\ $\mathrm{MD}, \mathrm{MPH}^{\top}$ \\ ${ }^{1}$ Center for Behavioral Cardiovascular Health, Columbia University Medical Center, New York, NY, USA; ${ }^{2}$ Case Western Reserve University, \\ Cleveland, OH, USA; ${ }^{3}$ The New York State Psychiatric Institute, Research Foundation for Mental Hygiene, New York, NY, USA; ${ }^{4}$ Department of \\ Psychiatry, Columbia University, College of Physicians and Surgeons, New York, NY, USA; ${ }^{5}$ New York Presbyterian Hospital, New York, NY, USA.
}

INTRODUCTION: Nearly 50\% of depressed primary care patients referred to mental health services do not initiate mental health treatment. The most promising interventions for increasing depression treatment initiation in primary care settings remain unclear.

METHODS: We performed a systematic search of publicly available databases from inception through August 2017 to identify interventions designed to increase depression treatment initiation. Two authors independently selected, extracted data, and rated risk of bias from included studies. Eligible studies used a randomized or pre-post design and assessed depression treatment initiation (i.e., $\geq 1$ mental health visit or antidepressant fill) among adults, the majority of whom met criteria for depression. Interventions were classified as simple or complex and subclassified into intervention strategies that were graded for strength of evidence.

RESULTS: Of 9516 articles identified, we included 14 unique studies representing 16 (4 simple and 12 complex) interventions and 8 treatment initiation strategies. We found low to moderate strength of evidence for collaborative/integrated care (3 studies), treatment preference matching ( 2 studies), and case management (2 studies) strategies. However, there was insufficient evidence to determine the benefit of cultural tailoring ( 2 studies), motivation (alone, with reminders or with cultural tailoring (5 studies)), education (1 study), and shared decision-making strategies (1 study). Overall, we found moderate strength of evidence for complex interventions (8 of 12 complex interventions demonstrated statistically significant effects on treatment initiation).

DISCUSSION: Collaborative/integrated care, preference treatment matching, and case management strategies had the best evidence for improving depression treatment initiation, but none of the strategies had high strength of evidence. While primary care settings can consider using some of these strategies when referring depressed

Electronic supplementary material The online version of this article (https://doi.org/10.1007/s11606-018-4554-z) contains supplementary material, which is available to authorized users.

Received January 30, 2018

Revised April 26, 2018

Accepted June 25, 2018

Published online August 14, 2018 patients to treatment, our review highlights the need for further rigorous research in this area.

KEY WORDS: depression; patient engagement/participation; primary healthcare.

J Gen Intern Med 33(11):1978-89

DOI: $10.1007 / \mathrm{s} 11606-018-4554-\mathrm{Z}$

(c) Society of General Internal Medicine 2018

\section{INTRODUCTION}

Between 5 and $13 \%$ of primary care patients carry a diagnosis of depression, ${ }^{1-3}$ contributing to morbidity and mortality. ${ }^{4-10}$ Numerous efficacious treatments exist, ${ }^{11-13}$ but nearly four in five depressed individuals worldwide fail to receive minimally adequate treatment. ${ }^{14}$ Depression is under-recognized in primary care, contributing to low treatment uptake. ${ }^{13,15}$ Yet, even when recognized, half of referred patients fail to attend psychotherapy visits ${ }^{16}$ or fill their first prescribed antidepressant medication. Even collaborative care programs, a team-based approach to delivering depression treatment in primary care settings, face $50 \%$ no show rates for initial visits with depression care managers. ${ }^{17-19}$

Lack of depression treatment initiation in primary care, the de facto location of depression care for most adults, ${ }^{20}$ is concerning. ${ }^{9}$ The process of engagement in depression treatment is a continuum of behaviors from intention to initiation to retention. Initiation is associated with treatment completion: as high as $40-60 \%$ of those who initiate therapy receive minimaly adequate treatment or complete a course according to some studies. ${ }^{14,21}$ Prior reviews assessing mental health engagement interventions have focused on patient activation, attitudes, or communication strategies $^{22,23}$ or included patients with mental illnesses other than depression. ${ }^{23,24}$ Few systematic reviews have sought to identify interventions for increasing treatment initiation $(\geq 1$ mental health visit or antidepressant prescription fill), ${ }^{25}$ particularly in depressed primary care populations. The purpose of this review is to identify interventions applicable to primary care settings that increase depression treatment initiation. 


\section{METHODS}

\section{Search Strategy}

This systematic review adheres to the Preferred Reporting Items for Systematic Reviews and Meta-Analyses statement (PRISMA; Fig. 1), and the protocol was published on PROSPERO (CRD42015026375). We searched Ovid MEDLINE, EMBASE, The Cochrane Library, CINAHL, and PsycINFO (Online Supplemental Table 1) for articles published from database inception to August 2017 to identify interventions seeking to increase depression treatment initiation. Search syntax was developed in consultation with an information specialist (LF) and comprised all relevant subject headings and free text terms used to define depression, randomized controlled trials (RCTs), clinical trials, and treatment initiation. We identified additional articles by reviewing reference lists of relevant reviews and studies and by utilizing the Similar Articles feature in PubMed and the Cited Reference Search in Scopus. Sources of gray literature (e.g., OpenGrey database) were searched as well as registries of ongoing trials, dissertations, and conference abstracts. Eligible designs included RCTs or pre-post design studies.

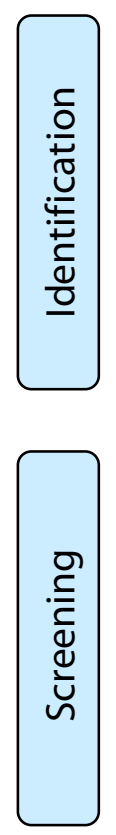

Records identified through database searching $(n=13,254)$
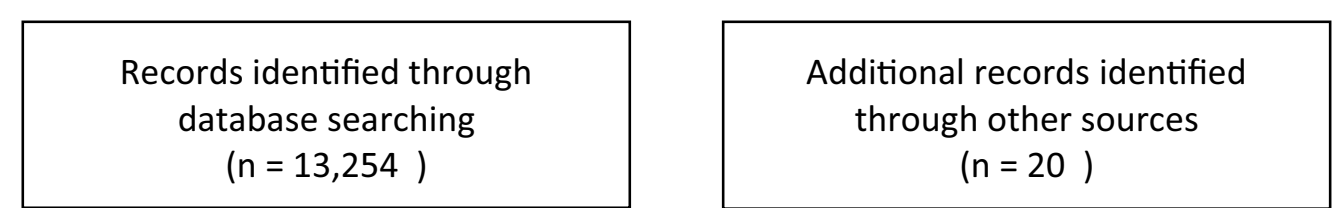

Records excluded ( $n=9,360$ )
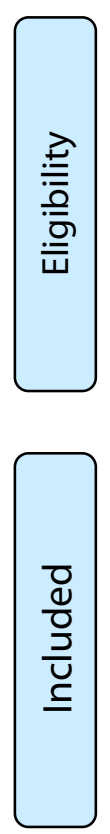

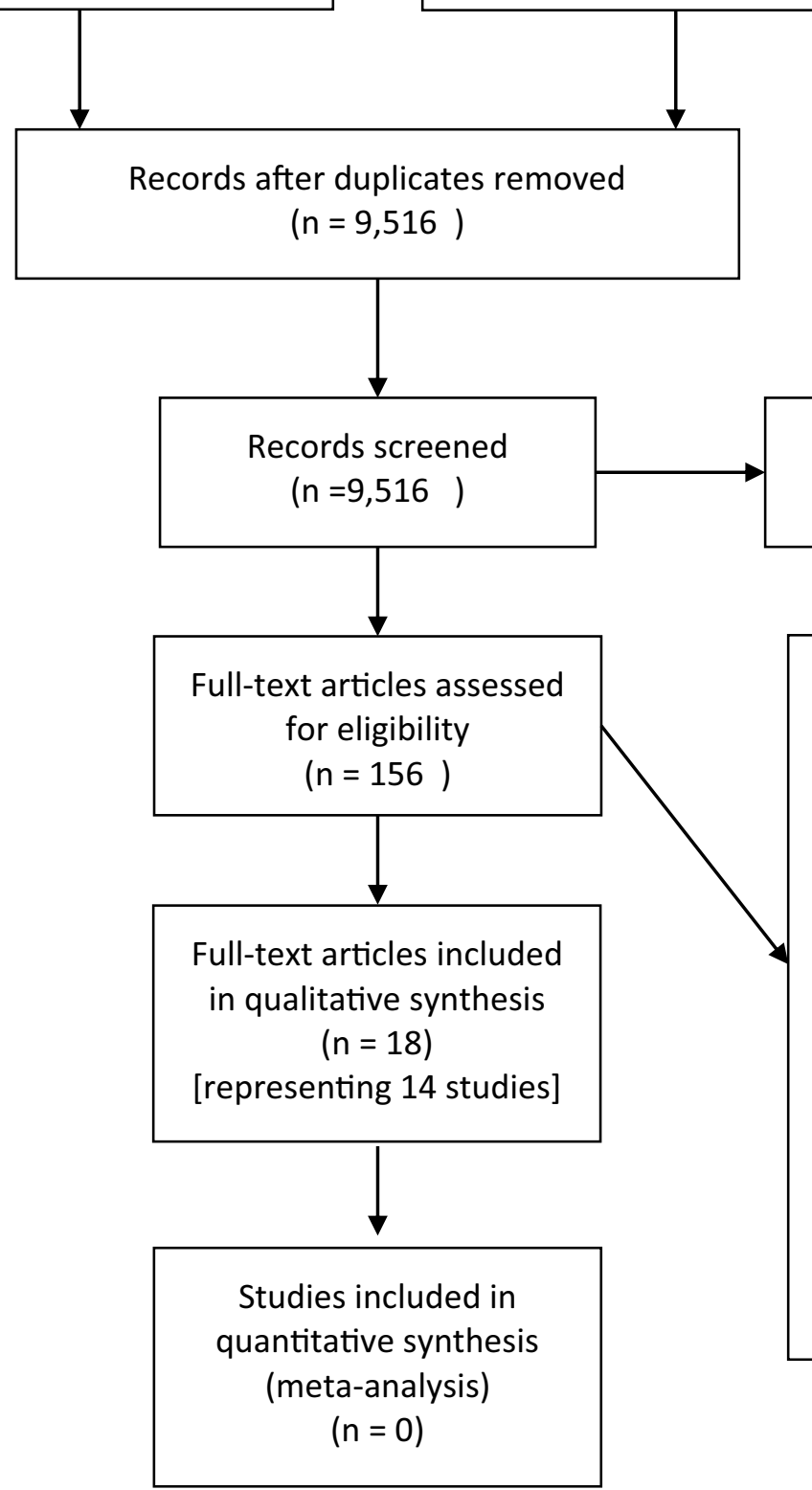

Full-text articles excluded, with reasons ( $n=142$ 26 Wrong study design 61 Wrong outcomes 27 Wrong population 17 Waiting for results, or additional publications 3 Wrong comparator 2 Language 1 Pediatric population 1 Wrong intervention 2 Wrong setting 2 depression threshold unknown) 


\section{Study Selection}

Study inclusion criteria were (1) participants $\geq 18$ years old; (2) relevant to primary care defined as interventions occurring in primary care, mixed primary and mental health, or community settings, including mental health settings with levels of complexity or contexts applicable to primary care and treatment initiation (e.g., a mental health clinic assessed the effect of a culturally tailored mental health booklet on treatment entry among patients scheduled for first time visits); (3) RCTs or pre-post study designs; and (4) depression treatment initiation outcome defined as attending $\geq 1$ visit with a mental health specialist (including depression care managers, defined as social workers or nurses tasked with directly providing psychotherapy or managing medications) or filling $\geq 1$ antidepressant prescription. ${ }^{25}$ We excluded studies (1) with participants < 18 years old; (2) not designed to increase treatment initiation; (3) non-English language publication; (4) mental health settings targeting patients already in care; and (5) targeting a population in which $<60 \%$ of participants had clinical diagnoses of depression or elevated depressive symptoms on a screening tool. To adhere to a pragmatic approach, we included studies seeking to increase treatment initiation in populations with mixed psychiatric diagnoses so long as a majority of the study population had depression. We contacted authors to clarify the study population when unclear. Our information specialist (LF) conducted the initial database search; then, two authors independently screened titles and abstracts for relevant papers with discrepancies resolved by consensus with a third author (LF, CG, and NM) within Covidence software (Veritas Health Innovation, Melbourne, Australia). Two authors independently reviewed full text copies of the relevant abstracts and titles using pre-defined eligibility criteria, with a third author available to resolve discrepancies (LF, MO, NM).

\section{Data Extraction}

We developed a standardized data extraction form to ensure uniformity. Two authors independently extracted information on study characteristics (LF, MO), including study location; study design; eligibility criteria; depression assessment method; intervention components; follow-up time; number of participants enrolled (total, intervention, and control); and demographic characteristics (sex, age, race, ethnicity). We categorized interventions as simple or complex based on the number, difficulty or variability of interacting components, behaviors required by those delivering or receiving the intervention, groups or organizational levels, and outcomes and degree of flexibility or tailoring permitted. ${ }^{26-28}$ Simple interventions had simple linear pathways between the intervention and outcome. ${ }^{28}$ In addition, we categorized interventions as patient, provider, system, or multilevel based on level of randomization and intervention. ${ }^{29}$ Discrepancies in data extraction were resolved through consensus with another reviewer (IK).

\section{Outcome Measure}

The primary outcome of interest was depression treatment initiation (i.e., attendance at $\geq 1$ appointment with a mental health specialist or self-reported or objective antidepressant initiation such as pharmacy fill of first prescription (i.e., primary adherence)). The secondary outcome measures, if available, were treatment retention (e.g., number of visits, proportion of days covered by an antidepressant medication as calculated from refill data) ${ }^{30}$ and mean change in depressive symptoms.

\section{Assessment of Risk of Bias}

Two authors independently assessed risk of bias utilizing the Cochrane Risk of Bias Tool for RCTs and the Quality Assessment Tool for Quantitative Studies for observational trials $(\mathrm{MO}, \mathrm{LF}) .^{31,32}$ We reviewed related method papers or contacted study authors when inadequate details were provided. Any discrepancies were resolved by consensus.

\section{Data Analysis and Synthesis}

Due to clinical and methodological heterogeneity and low numbers of similar studies, we decided post hoc not to conduct pooled quantitative analyses and synthesized data qualitatively. Two coders (NM, MO) independently grouped interventions into categories that reflected key intervention components (isolated from controls), using a previously developed classification model for engagement strategies that focuses on information, activation, and collaboration at patient and provider levels, ${ }^{33}$ and guided by prior systematic reviews on behavioral interventions. ${ }^{34}$ We enlisted one author to achieve consensus (IK). "Motivation" strategies included motivational interviewing, patient activation, or other behavioral interventions (i.e., goal setting). Finally, we graded the strength of evidence (SOE) for each intervention strategy on treatment initiation, retention, medication adherence, and depressive symptoms per an Agency for Healthcare Research and Quality and Effective Healthcare Program protocol; the grade incorporated four key considerations to determine strength of a stated effect: risk of bias (including study design and aggregate quality), consistency, direction, and precision across studies testing a particular strategy or intervention type (i.e., simple or complex) on a particular outcome (e.g., initiation). ${ }^{35}$

\section{RESULTS}

Initial database search yielded 9516 unique references. Subsequent screen of the titles and abstracts for relevant papers resulted in 156 potentially relevant papers for full text review and 18 papers that met inclusion criteria representing 14 unique studies in this review (Fig. 1, Online Supplemental Table 2). ${ }^{25,30,36-51}$ We excluded two studies because they included mixed psychiatric disorders but no description of whether the majority of participants were depressed. ${ }^{52,53}$ 


\section{Study Characteristics}

Studies were published between 2000 and 2016, with the majority $(n=12)$ conducted in the USA. Of the 14 unique studies, 4 recruited patients from community settings (e.g., electoral roll, managed care beneficiaries), ${ }^{42,44,45,50} 2$ recruited patients from both primary care and mental health clinics, ${ }^{38,40,41,46} 6$ from primary care or outpatient or community health settings, ${ }^{48}$ and 2 from mental health clinics with interventions highly applicable to the primary care setting. ${ }^{36,49}$ Sample sizes ranged from 42 to 2022 participant; mean participant age ranged from 35 to 83 years. Depression diagnosis eligibility was determined using a structured interview $(n=4),{ }^{36,37,39,43,47,50}$ validated depression screening tools (e.g., Center for Epidemiologic Studies Depression Scale [CESD] or Patient Health Questionnaire [PHQ]-9) $(n=7),{ }^{30,38,40,41,44,46,48,49,51}$ a combination of interview and validated screening tool $(n=2),{ }^{25,45}$ and psychological distress tools $(n=1)$ (Table 1). ${ }^{42}$

There was 1 pre-post design, 1 pre-post design within an $\mathrm{RCT},{ }^{46,48}$ and the remainder were RCTs. ${ }^{25,30,36-47,49-51}$ For studies presenting multiple follow-up periods, we used the minimum follow-up time to isolate intervention effect on treatment initiation. The minimum follow-up time ranged from 4 to 48 weeks; 2 did not clearly report follow-up times (Table 1). ${ }^{48,49}$

\section{Outcome Measures}

There was some variability in the definition of treatment initiation. Studies reported $\geq 1$ therapy visits (mental health, psychiatry, psychology or counseling visits; $n=$ 7), ${ }^{36,38,40,41,49,46,48,50,51}$ medication use $(n=1),{ }^{30}$ a composite of therapy or medication $(n=1),{ }^{25}$ or separately reported therapy and medication $(n=4) .^{37-42,44}$ One study comparing the effect of treatment preference matching vs. mismatching $(n=1)$ used refusal of randomization after participant notification of study arm as a proxy for treatment non-initiation (a primary outcome). ${ }^{45}$ Treatment retention outcomes included percent of visits attended, ${ }^{45}$ mean number of visits attended, ${ }^{34,36,38,40,41,49}$ proportion of days covered (PDC) by antidepressant, ${ }^{30,34}$ and treatment completion (Table 1). ${ }^{44}$ Change in depression symptoms was assessed as an outcome by 8 trials (Online Supplemental Table 2).

\section{Risk of Bias}

We report risk of bias for all 18 papers representing 14 unique studies (Fig. 2, Online Supplemental Fig. 1). Overall, the prepost trial by Lara (2003) had a weak global rating ${ }^{46}$ according to the Quality Assessment Tool for Quantitative Studies. ${ }^{31}$ Of the remaining RCTs, only one had low risk of bias ${ }^{39}$ according to the Cochrane tool. Seven did not clearly report a randomization method. ${ }^{25,36,38,41,43,47,50}$ All displayed moderate to high bias related to blinding participants or personnel and 5 clearly reported blinding of outcome assessors (Fig. 2, Online Supplemental Fig. 1).

\section{Effect of Interventions on Outcomes}

Overall, 14 studies assessed 16 interventions (i.e., 2 studies assessed 2 interventions) ${ }^{42,51}$ comprising 4 simple and 12 complex interventions. Overall, 2 of 4 (50\%) simple interventions and 8 of $12(67 \%)$ complex interventions reported a statistically significant difference in depression treatment initiation between intervention and control. By definition, simple interventions were patient level while complex interventions comprised both patient and multi- (i.e., system or provider and patient) levels; 6 of 11 patient-level interventions (55\%) reported statistically significant differences compared to 4 of 5 multilevel (80\%) interventions ${ }^{30,37-41,43,47,48}$ (Table 1).

Our qualitative analyses identified 8 distinct treatment initiation strategies: case management, collaborative/integrated care, cultural tailoring, education, motivation, motivation and reminders, motivation and cultural tailoring, and treatment preference matching. Case management sub-strategies included appointment facilitation, motivation, and education with or without preference matching. Collaborative/integrated care sub-strategies included preference matching and onsite access with or without motivation and education. Below, we provide a summary of strategies organized by simple or complex interventions.

\section{Simple Interventions}

Patient Level. Cultural Tailoring. There was no difference in treatment entry between Black patients randomized to receiving targeted educational material about mental health and stigma and those receiving general mental health information (Table 1). ${ }^{36}$

Motivation. Delgadillo's (2015) mailed theory-based orientation leaflet addressing expectations and barriers did not significantly differ from a mailed appointment confirmation in improving low-intensity cognitive behavioral therapy (CBT) initiation (48/81 (54\%) vs. 60/91 (66\%)) (Table 1).

Treatment Preference Matching. Kwan (2010) and Raue (2009) matched (vs. mismatched) treatment allocation and preference (therapy or antidepressants), demonstrating significantly improved initiation of assigned treatment that matched preference $\left(26 / 26(100 \%)\right.$ vs. $37 / 44(84 \%)^{45}$ and $29 / 29(100 \%)$ vs. $23 / 31(74 \%)) .{ }^{25}$ Kwan (2010) demonstrated improvement in proportion of therapy visits attended and dropout rates, but neither study found significant improvement in depressive symptoms (Table 1, Online Supplemental Table 2).

\section{Complex Interventions}

Patient Level. Case Management. Kim (2011) found that a telephone case management outreach program that facilitated appointments, referrals, provided appointment reminders, education, engagement, and monitoring of progress for 
Table 1 Study Characteristics for Systematic Review of Depression Treatment Initiation Interventions

\begin{tabular}{|c|c|c|c|c|c|c|c|c|}
\hline$\overline{\text { Study }}$ & $\begin{array}{l}\text { Theme (level)* } \\
\text { (main theme } \\
\text { and } \\
\text { subthemes } \\
\text { isolated } \\
\text { compared } \\
\text { to control) }\end{array}$ & 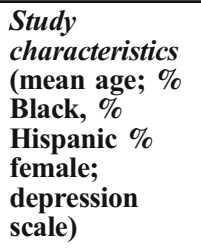 & $\begin{array}{l}\text { Setting } \\
\text { (follow-up } \\
\text { time for } \\
\text { outcome) }\end{array}$ & $\begin{array}{l}\text { Intervention vs. } \\
\text { Control }\end{array}$ & $\begin{array}{l}\text { Intervention } \\
\text { outcome, } \\
\text { frequency } \\
(\%)\end{array}$ & $\begin{array}{l}\text { Control } \\
\text { outcome, } \\
\text { frequency } \\
(\%)\end{array}$ & $\begin{array}{l}\text { Effect } \\
\text { size, OR } \\
(95 \% \text { CI) }\end{array}$ & 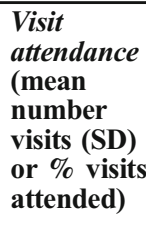 \\
\hline \multicolumn{9}{|c|}{ Simple interventions (Boldface if $p<0.05$ ) } \\
\hline $\begin{array}{l}\text { Alvidrez } \\
\text { (2009) }\end{array}$ & $\begin{array}{l}\text { Cultural } \\
\text { tailoring }\end{array}$ & $\begin{array}{l}44.8 \text { years; } \\
100 \% \mathrm{~B} ; 69 \% \mathrm{~F} \\
\text { DSMIV }\end{array}$ & $\begin{array}{l}\text { Mental } \\
\text { health clinic } \\
\text { (12 weeks) }\end{array}$ & $\begin{array}{l}\text { Targeted } \\
\text { educational } \\
\text { booklet vs. } \\
\text { general } \\
\text { information }\end{array}$ & $\begin{array}{l}\text { Psych visit } \\
17 / 22(76 \%)\end{array}$ & $\begin{array}{l}\text { Psych visit } \\
14 / 20(71 \%)\end{array}$ & NR & 4.9 vs. 4.6 \\
\hline $\begin{array}{l}\text { Delgadillo } \\
\text { (2015) }\end{array}$ & Motivation & $\begin{array}{l}40 \text { years, } \\
58 \% \mathrm{~F}, 40.6 \\
\text { (14.9) years; } \\
\text { PHQ9 }\end{array}$ & $\begin{array}{l}\text { Primary care } \\
\text { IAPT, } \\
\text { England } \\
\text { (6 weeks) }\end{array}$ & $\begin{array}{l}\text { Orientation } \\
\text { leaflet on } \\
\text { expectations, } \\
\text { normalize } \\
\text { concerns vs. } \\
\text { mailed } \\
\text { confirmation }\end{array}$ & $\begin{array}{l}\text { Psych visit } \\
44 / 81(54 \%)\end{array}$ & $\begin{array}{l}\text { Psych visit } \\
60 / 91(66 \%)\end{array}$ & $\begin{array}{l}\mathrm{B}=-0.18 \\
(0.85) \\
\mathrm{AOR}= \\
0.85\end{array}$ & $\begin{array}{l}\text { Complete } \\
58 \% \text { vs. } \\
71 \% ; \\
N=\text { NR }\end{array}$ \\
\hline $\begin{array}{l}\text { Kwan } \\
(2010)\end{array}$ & $\begin{array}{l}\text { Treatment } \\
\text { preference } \\
\text { matching }\end{array}$ & $\begin{array}{l}38.4 \text { years; } \\
4 \% \mathrm{~B} ; 7 \% \mathrm{H} ; \\
64 \% \mathrm{~F} ; \\
\text { DSMIV/BDI- } \\
\text { II/HRSD }\end{array}$ & $\begin{array}{l}\text { Community } \\
\text { (telephone } \\
\text { screening) } \\
\text { (16 weeks) }\end{array}$ & $\begin{array}{l}\text { Match to } \\
\text { preference for } \\
\text { meds or therapy } \\
\text { vs. mismatch vs. } \\
\text { no preference }\end{array}$ & $\begin{array}{l}\text { Any } T x \\
26 / 26 \\
(100 \%)^{\dagger}\end{array}$ & $\begin{array}{l}\text { Any Tx } \\
37 / 44 \\
(84 \%)^{\dagger} \\
\text { vs. } \\
31 / 36 \\
(86 \%)^{\dagger}\end{array}$ & $\begin{array}{l}X^{2}=4.60 \\
p=0.03 \\
\text { NR }\end{array}$ & $\begin{array}{l}\text { \% visits: } \\
\mathbf{8 9 \%} \text { vs. } \\
\mathbf{7 0 \%} \\
\text { vs. } 85 \%\end{array}$ \\
\hline $\begin{array}{l}\text { Raue } \\
(2009)\end{array}$ & $\begin{array}{l}\text { Treatment } \\
\text { preference } \\
\text { matching }\end{array}$ & $\begin{array}{l}51.2 \text { years; } \\
20 \% \mathrm{~B} ; 40 \% \\
\mathrm{H} ; 78 \% \mathrm{~F} ; \\
\text { DSMIV/HRSD }\end{array}$ & $\begin{array}{l}\text { Ambulatory } \\
\text { care clinics } \\
\text { (12 weeks) }\end{array}$ & $\begin{array}{l}\text { Match to } \\
\text { preference for } \\
\text { meds or therapy } \\
\text { vs. mismatch }\end{array}$ & $\begin{array}{l}\text { Any Tx } \\
29 / 29 \\
(100 \%)\end{array}$ & $\begin{array}{l}\text { Any Tx } \\
23 / 31(74 \%)\end{array}$ & $\begin{array}{l}O R=5.3 \\
(4.3-6.3)\end{array}$ & $\begin{array}{l}\text { Reported } \\
\text { as "Null" }\end{array}$ \\
\hline \multicolumn{9}{|c|}{ Complex interventions } \\
\hline $\begin{array}{l}\text { Kim } \\
(2011)\end{array}$ & $\begin{array}{l}\text { Case } \\
\text { management } \\
\text { (appointment, } \\
\text { education, } \\
\text { motivation, } \\
\text { reminder) }\end{array}$ & $\begin{array}{l}35 \text { years; } \\
12 \% \mathrm{~B} ; 33 \% \\
\text { H; 90\%F; } \\
\text { K6/QIDS-SR }\end{array}$ & $\begin{array}{l}\text { Medicaid } \\
\text { managed } \\
\text { care } \\
\text { beneficiaries } \\
\text { (48 weeks) }\end{array}$ & $\begin{array}{l}\text { Phone calls } \\
\text { to facilitate } \\
\text { appointments, } \\
\text { engage, } \\
\text { educate vs. } \\
\text { mailed mental } \\
\text { health provider } \\
\text { list }\end{array}$ & $\begin{array}{l}\text { Any Tx 42/ } \\
234(19 \%)^{*} \\
\text { Psychiatry } \\
18 / 234 \\
(12 \%) \text { Psy- } \\
\text { chology } \\
30 / 234 \\
(15 \%) \text { Med } \\
53 / 234 \\
(27 \%)\end{array}$ & $\begin{array}{l}\text { Any Tx 31/ } \\
242(17 \%)^{\ddagger} \\
\text { Psychiatry } \\
10 / 242(7 \%) \\
\text { Psychology } \\
20 / 242 \\
(12 \%) \mathrm{Med} \\
42 / 242(27 \% \\
\text { reported) }\end{array}$ & $\begin{array}{l}\mathrm{OR}=1.51 \\
(1.00- \\
2.28) \\
\text { OR=1.90 } \\
(\mathbf{1 . 0 8}- \\
\mathbf{3 . 3 5 )} \\
\mathbf{O R}=\mathbf{1 . 6 6} \\
(\mathbf{1 . 0 6 -} \\
\mathbf{2 . 6 1 )} \\
\mathrm{OR}=1.34 \\
(0.88- \\
2.11)\end{array}$ & $\begin{array}{l}3.6(2.2) \\
\text { vs. } 2.1 \\
(2.0)\end{array}$ \\
\hline $\begin{array}{l}\text { Sirey } \\
(2016)\end{array}$ & $\begin{array}{l}\text { Case } \\
\text { management } \\
\text { (appointment, } \\
\text { motivation, } \\
\text { preference) }\end{array}$ & $\begin{array}{l}\text { Intervention: } \\
82.9 \text { years; } \\
61 \% \mathrm{~F} ; 21 \% \mathrm{~B} \\
\text { control: } 81.0 \\
\text { years; } 82 \% \mathrm{~F} ; \\
31 \% \mathrm{~B} \text { SCID } \\
\text { DSMIV }\end{array}$ & $\begin{array}{l}\text { Homebound } \\
\text { community } \\
\text { dwelling } \\
\text { ( } 24 \text { weeks) }\end{array}$ & $\begin{array}{l}\text { Refer acc/ } \\
\text { preference, cost; } \\
\text { assess barriers, } \\
\text { goals; MI; } \\
\text { educate vs. } \\
\text { recommend } \\
\text { provider and } \\
\text { educate } \\
\text { ( } 6 \text { home visits } \\
\text { and } 2 \text { calls) }\end{array}$ & $\begin{array}{l}\text { Any Tx } \\
60 / 81 \\
(74 \%)^{*}\end{array}$ & $\begin{array}{l}\text { Any Tx } \\
45 / 80 \\
(56 \%)^{*}\end{array}$ & $\begin{array}{l}\text { AOR }= \\
2.4(1.17- \\
4.93)\end{array}$ & NR \\
\hline
\end{tabular}

(continued on next page)

Medicaid-managed care beneficiaries (vs. providing a list of behavioral providers) did not significantly improve receipt of any mental services or antidepressant use or depressive symptoms but did improve psychiatry visits $(18 / 234$ (12\%) vs. 10/ $242(7 \%) ; \mathrm{OR}=1.90,95 \% \mathrm{CI} 1.08-3.35)$ and mean number of visits (Table 1; Online Supplemental Table 2). ${ }^{44}$ Sirey (2016) evaluated a case management program for elderly depressed adults qualifying for a home meal program, which focused on patient preference matching, patient activation, education, and treatment options. Both intervention and attention control arms received 6 in-home visits and 2 follow-up calls (60/81 $(74 \%)$ vs. $45 / 80(56 \%), \mathrm{OR}=2.4095 \% \mathrm{CI} 1.17-4.93]) .{ }^{50}$
Motivation/Education. An Internet-delivered CBT and activation intervention (vs. Internet-delivered depression literacy; education) both accompanied by weekly calls $\times 5$ weeks (vs. attention control phone calls only) improved reported CBT use $(35 / 121(29 \%)$ vs. $15 / 136(11 \%)$ vs. $12 / 157(8 \%))$ but not medication use or counselor or psychologist help seeking $(18 \%$ vs. $10 \%[\mathrm{OR}=1.93,95 \% \mathrm{CI}=0.94-3.98]$ vs. $16 \%)$ (Table 1). ${ }^{30}$ By consensus, we categorized this as "motivation" and as effective, though the site may have improved perception of receiving online CBT (via access and reminders) but not help seeking behavior. Both internetdelivered motivation and education significantly improved 
Table 1. (continued)

\begin{tabular}{|c|c|c|c|c|c|c|c|c|}
\hline$\overline{\text { Study }}$ & $\begin{array}{l}\text { Theme (level)* } \\
\text { (main theme } \\
\text { and } \\
\text { subthemes } \\
\text { isolated } \\
\text { compared } \\
\text { to control) }\end{array}$ & 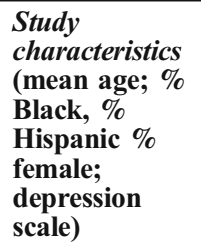 & $\begin{array}{l}\text { Setting } \\
\text { (follow-up } \\
\text { time for } \\
\text { outcome) }\end{array}$ & $\begin{array}{l}\text { Intervention vs. } \\
\text { Control }\end{array}$ & $\begin{array}{l}\text { Intervention } \\
\text { outcome, } \\
\text { frequency } \\
(\%)\end{array}$ & $\begin{array}{l}\text { Control } \\
\text { outcome, } \\
\text { frequency } \\
(\%)\end{array}$ & $\begin{array}{l}\text { Effect } \\
\text { size, OR } \\
\text { (95\% CI) }\end{array}$ & 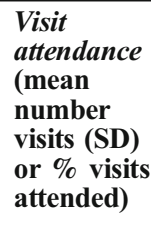 \\
\hline $\begin{array}{l}\text { Arean } \\
(2005)^{\S} \\
\text { Arean } \\
(2007)\end{array}$ & $\begin{array}{l}\text { Collaborativel } \\
\text { integrated } \\
\text { care } \\
\text { (education, } \\
\text { motivation, } \\
\text { preference, } \\
\text { onsite) (mult- } \\
\text { level) }\end{array}$ & $\begin{array}{l}71.2 \text { years; } \\
12 \% \mathrm{~B} ; 8 \% \mathrm{H} ; \\
65 \% \mathrm{~F} ; \mathrm{SCID} \\
\text { DSMIV }\end{array}$ & $\begin{array}{l}\text { Primary care } \\
\text { clinic } \\
\text { (IMPACT } \\
\text { Trial) } \\
\text { (12 weeks) }\end{array}$ & $\begin{array}{l}\text { Collaborative } \\
\text { care vs. usual } \\
\text { care }\end{array}$ & $\begin{array}{l}\text { Any Tx } \\
\text { Not poor } \\
500 / 627 \\
\text { (80\%) } \\
\text { Poor } \\
208 / 279 \\
\text { (75\%) Psych } \\
\text { visit and } \\
\text { Med" }\end{array}$ & $\begin{array}{l}\text { Any Tx } \\
\text { Not poor } \\
319 / 598 \\
\text { (54\%) } \\
\text { Poor } \\
162 / 297 \\
\text { (55\%) Psych } \\
\text { visit and } \\
\text { Med" }\end{array}$ & $\begin{array}{l}\text { AOR = } \\
4.18 \\
(3.09- \\
5.65) \\
\text { AOR= } \\
2.99 \\
(2.02- \\
4.44)\end{array}$ & NR \\
\hline $\begin{array}{l}\text { Bartels } \\
(2004), \\
\text { Ayalon } \\
(2007)^{\S}, \\
\text { Arean } \\
(2008)\end{array}$ & $\begin{array}{l}\text { Collaborative/ } \\
\text { integrated care } \\
\text { (onsite, } \\
\text { preference) } \\
\text { (multilevel) }\end{array}$ & $\begin{array}{l}73.5 \text { years; } \\
25 \% \mathrm{~B} ; 15 \% \mathrm{H} ; \\
26 \% \mathrm{~F} ; \\
\text { MINI/CESD }\end{array}$ & $\begin{array}{l}\text { VA primary } \\
\text { and mental } \\
\text { health (10 } \\
\text { PRISM-E } \\
\text { Study sites) } \\
\text { (24 weeks) }\end{array}$ & $\begin{array}{l}\text { Integrated Care } \\
\text { (onsite, expedite } \\
\text { appts, trained } \\
\text { specialist } \\
\text { communicates } \\
\text { with PMD } \pm \\
\text { brief alcohol } \\
\text { intervention) vs. } \\
\text { enhanced } \\
\text { referral (help } \\
\text { cost, transport, } \\
\text { expedite appts) }\end{array}$ & $\begin{array}{l}\text { Psych visit } \\
709 / 999 \\
(71 \%)\end{array}$ & $\begin{array}{l}\text { Psych visit } \\
499 / 1023 \\
(49 \%)\end{array}$ & $\begin{array}{l}\mathrm{OR}=2.57 \\
(\mathbf{2 . 1 4} \\
\mathbf{3 . 0 8})\end{array}$ & $\begin{array}{l}3.04(3.7) \\
\text { vs. } 1.91 \\
(3.6)\end{array}$ \\
\hline $\begin{array}{l}\text { Wells } \\
(2000), \\
\text { Jacoux } \\
(2003)\end{array}$ & $\begin{array}{l}\text { Collaborative/ } \\
\text { integrated care } \\
\text { (education, } \\
\text { motivation, } \\
\text { onsite, } \\
\text { preference) } \\
\text { (multilevel) }\end{array}$ & $\begin{array}{l}43.7 \text { years; } \\
7 \% \mathrm{~B} ; 29 \% \mathrm{H} ; \\
72 \% \mathrm{~F} ; \mathrm{CIDI}\end{array}$ & $\begin{array}{l}\text { Primary care } \\
\text { clinic } \\
\text { ( } 24 \text { weeks) }\end{array}$ & $\begin{array}{l}\text { Video; } \\
\text { pamphlets; } \\
\text { nurses and } \\
\text { providers trained } \\
\text { in activation, } \\
\text { therapy (QI } \\
\text { therapy) or med } \\
\text { management (QI } \\
\text { med) vs. usual } \\
\text { care }\end{array}$ & $\begin{array}{l}\text { Any Tx 51\% } \\
\text { (48\%-54\%) } \\
\text { Psych visit } \\
38 \%(35 \%- \\
41 \%) \text { Med } \\
35 \%(32 \%- \\
38 \%)(N= \\
913 ; \\
\text { adjusted \%, } \\
\text { frequencies } \\
N R)\end{array}$ & $\begin{array}{l}\text { Any Tx 40\% } \\
\text { (36-44\%) } \\
\text { Psych visit } \\
26 \% \\
(22-30 \%) \\
\text { Med 25\% } \\
(21-29 \%) \\
(N=443 ; \\
\text { adjusted \%, } \\
\text { frequencies } \\
\text { NR) }\end{array}$ & $\begin{array}{l}t=3.50 \\
p<0.001 \\
t=3.99 \\
p<0.001 \\
t=3.38 \\
p<0.001\end{array}$ & $\begin{array}{l}2.5 \text { vs. } \\
2.9 \text { vs. } \\
2.2\end{array}$ \\
\hline $\begin{array}{l}\text { Yeung } \\
(2010)\end{array}$ & $\begin{array}{l}\text { Cultural } \\
\text { tailoring } \\
\text { (multilevel) }\end{array}$ & $\begin{array}{l}49 \text { years; } 0 \% \mathrm{~B} ; \\
0 \% \mathrm{H} ; 68 \% \mathrm{~F} \\
100 \% \text { Chinese; } \\
\text { Chinese- } \\
\text { PHQ9, } \\
\text { HAMD17 }\end{array}$ & $\begin{array}{l}\text { Community } \\
\text { health center } \\
\text { (weeks N/A) }\end{array}$ & $\begin{array}{l}\text { Pre-post Chinese } \\
\text { PHQ9 and } \\
\text { culturally } \\
\text { tailored contact } \\
\text { and psychiatric } \\
\text { evaluation prior } \\
\text { to collaborative } \\
\text { care RCT }\end{array}$ & $\begin{array}{l}\text { Psych visit } \\
100 / 233 \\
(43 \%)\end{array}$ & $\begin{array}{l}\text { Psych visit } \\
19 / 296(7 \%)\end{array}$ & NR & NR \\
\hline \multirow[t]{2}{*}{$\begin{array}{l}\text { Christensen } \\
(2006)\end{array}$} & Education & $\begin{array}{l}36.8 \text { years; } \\
73 \% \mathrm{~F} ; \text { Kessler } \\
\text { Psychological } \\
\text { Distress Scale }\end{array}$ & $\begin{array}{l}\text { Community } \\
\text { electoral in } \\
\text { Australia } \\
\text { ( } 5 \text { weeks) }\end{array}$ & $\begin{array}{l}\text { 5-week } \\
\text { depression } \\
\text { information } \\
\text { website vs. } \\
\text { attention control } \\
\text { (both phoned) }\end{array}$ & $\begin{array}{l}\text { Psych visit } \\
15 / 136 \\
(11 \%)^{\#} \mathrm{Med} \\
38 / 136 \\
(28 \%)\end{array}$ & $\begin{array}{l}\text { Psych visit } \\
12 / 157 \\
(8 \%)^{\#} \mathrm{Med} \\
40 / 157 \\
(26 \%)\end{array}$ & $\begin{array}{l}\mathrm{OR}=1.50 \\
(0.68- \\
3.33) \\
\mathrm{OR}=1.14 \\
(1.89- \\
0.68)\end{array}$ & NR \\
\hline & Motivation & $\begin{array}{l}36.8 \text { years; } \\
73 \% \mathrm{~F} ; \text { Kessler } \\
\text { Psychological } \\
\text { Distress Scale }\end{array}$ & $\begin{array}{l}\text { Community } \\
\text { electoral in } \\
\text { Australia } \\
\text { ( } 5 \text { weeks) }\end{array}$ & $\begin{array}{l}\text { 5-week CBT } \\
\text { training vs. } \\
\text { depression } \\
\text { information } \\
\text { websites (both } \\
\text { phoned with } \\
\text { prompts) }\end{array}$ & $\begin{array}{l}\text { Psych visit } \\
35 / 121 \\
(29 \%)^{\#} \text { Med } \\
30 / 121 \\
(25 \%)\end{array}$ & $\begin{array}{l}\text { Psych visit } \\
15 / 136 \\
(11 \%)^{\#} \mathrm{Med} \\
38 / 136 \\
(26 \%)\end{array}$ & $\begin{array}{l}\text { OR=3.28 } \\
(\mathbf{1 . 6 9 -} \\
\mathbf{6 . 3 8 )} \\
\mathrm{OR}=1.85 \\
(0.49- \\
1.48)\end{array}$ & NR \\
\hline
\end{tabular}

(continued on next page)

CESD measured depressive symptoms compared to control (Mean difference: $-4.5[-7.3$ to -1.8$]$ and $-3.6[-6.3$ to 1.0], respectively) (Online Supplemental Table 2).

Motivation and Reminder. A second intervention by Delgadillo (2015) found that mailed theory-informed leaflets with text reminders $(56 / 82(68 \%))$ were no more effective than appointment confirmation (60/91 (66\%)) or leaflets alone (see simple motivation strategy above), with no significant effects on number of attended visits (Table 1; Online Supplemental Table 2). ${ }^{51}$ A trial involving a letter confirmation and phone reminder 
Table 1. (continued)

\begin{tabular}{|c|c|c|c|c|c|c|c|c|}
\hline Study & $\begin{array}{l}\text { Theme (level)* } \\
\text { (main theme } \\
\text { and } \\
\text { subthemes } \\
\text { isolated } \\
\text { compared } \\
\text { to control) }\end{array}$ & $\begin{array}{l}\text { Study } \\
\text { characteristics } \\
\text { (mean age; \% } \\
\text { Black, \% } \\
\text { Hispanic \% } \\
\text { female; } \\
\text { depression } \\
\text { scale) }\end{array}$ & $\begin{array}{l}\text { Setting } \\
\text { (follow-up } \\
\text { time for } \\
\text { outcome) }\end{array}$ & $\begin{array}{l}\text { Intervention vs. } \\
\text { Control }\end{array}$ & $\begin{array}{l}\text { Intervention } \\
\text { outcome, } \\
\text { frequency } \\
(\%)\end{array}$ & $\begin{array}{l}\text { Control } \\
\text { outcome, } \\
\text { frequency } \\
(\%)\end{array}$ & $\begin{array}{l}\text { Effect } \\
\text { size, OR } \\
(95 \% \text { CI })\end{array}$ & $\begin{array}{l}\text { Visit } \\
\text { attendance } \\
\text { (mean } \\
\text { number } \\
\text { visits (SD) } \\
\text { or \% visits } \\
\text { attended) }\end{array}$ \\
\hline Lara (2003) & $\begin{array}{l}\text { Motivation and } \\
\text { cultural } \\
\text { tailoring }\end{array}$ & $\begin{array}{l}35.3 \text { years; } \\
100 \% \mathrm{~F} ; \mathrm{CESD} \text {, } \\
\text { DSMIII }\end{array}$ & $\begin{array}{l}\text { Primary/ } \\
\text { mental } \\
\text { health, } \\
\text { Mexico } \\
\text { (16 weeks) }\end{array}$ & $\begin{array}{l}\text { Pre-post } 6 \text { group } \\
\text { sessions } \\
\text { (education, } \\
\text { activation, self- } \\
\text { management, } \\
\text { culture and } \\
\text { gender } \\
\text { tailored) vs. } \\
\text { 20-min } \\
\text { explanation and } \\
\text { educational } \\
\text { material }\end{array}$ & $\begin{array}{l}\text { Psych visit: } \\
23 / 107 \\
(22 \%)(N= \\
179 \text { pre- } \\
\text { intervention; } \\
N=107 \text { post })\end{array}$ & $\begin{array}{l}\text { Psych visit: } \\
8 / 47(17 \%) \\
(N=75 \text { pre- } \\
\text { intervention; } \\
N=47 \text { post) }\end{array}$ & $\begin{array}{l}X^{2}= \\
0.176 \\
p=0.67\end{array}$ & NR \\
\hline $\begin{array}{l}\text { Zanjani } \\
(2008)\end{array}$ & $\begin{array}{l}\text { Motivation and } \\
\text { reminder }\end{array}$ & $\begin{array}{l}53 \text { years; } \\
63 \% \mathrm{~B} ; 3 \% \mathrm{H} ; \\
4 \% \mathrm{~F} \text {; PHQ9 }\end{array}$ & $\begin{array}{l}\text { VA primary } \\
\text { care confirm } \\
\text { eligible by } \\
\text { behavioral } \\
\text { health lab }\end{array}$ & $\begin{array}{l}1-2 \text { brief } \\
\text { motivational and } \\
\text { reminder call by } \\
\text { behavior } \\
\text { specialist, letter, } \\
\text { and auto } \\
\text { reminder vs. } \\
\text { letter and auto } \\
\text { reminder }\end{array}$ & $\begin{array}{l}\text { Psych visit } \\
40 / 57(70 \%)\end{array}$ & $\begin{array}{l}\text { Psych visit } \\
18 / 56(32 \%)\end{array}$ & $\begin{array}{l}X^{2}=0.16 \\
p<0.001\end{array}$ & $\begin{array}{l}3 \text { (3) vs. } \\
2(3)\end{array}$ \\
\hline $\begin{array}{l}\text { Delgadillo } \\
(2015)\end{array}$ & $\begin{array}{l}\text { Motivation and } \\
\text { reminder }\end{array}$ & $\begin{array}{l}40 \text { years, } \\
58 \% \mathrm{~F}, 40.6 \\
\text { (14.9) years; } \\
\text { PHQ9 }\end{array}$ & $\begin{array}{l}\text { Primary care } \\
\text { IAPT, } \\
\text { England } \\
\text { ( } 6 \text { weeks) }\end{array}$ & $\begin{array}{l}\text { Theory-based } \\
\text { orientation } \\
\text { leaflet on } \\
\text { expectations and } \\
\text { normalize } \\
\text { concerns and } \\
\text { 48-h text } \\
\text { reminder vs. } \\
\text { mailed } \\
\text { confirmation }\end{array}$ & $\begin{array}{l}\text { Psych visit } \\
56 / 82(68 \%)\end{array}$ & $\begin{array}{l}\text { Psych visit } \\
60 / 91(66 \%)\end{array}$ & $\begin{array}{l}B=-0.06 \\
(0.72) \\
\text { AOR }= \\
0.95\end{array}$ & $\begin{array}{l}\text { Complete } \\
\text { Tx } 74 \text { vs. } \\
71 \% \\
N=\text { NR }\end{array}$ \\
\hline $\begin{array}{l}\text { Le Blanc } \\
(2016)\end{array}$ & $\begin{array}{l}\text { Shared or } \\
\text { clinical } \\
\text { decision- } \\
\text { making and } \\
\text { education } \\
\text { (multilevel) }\end{array}$ & $\begin{array}{l}\text { Intervention } \\
43.2 \text { years; } \\
72 \% \text { F control } \\
43.9 \text { years; } \\
62 \% \mathrm{~F} \text {; PHQ9 }\end{array}$ & $\begin{array}{l}\text { Primary } \\
\text { care; cluster } \\
\text { RCT of } \\
\text { clinicians } \\
\text { and patients } \\
\text { ( } 4 \text { weeks) }\end{array}$ & $\begin{array}{l}\text { Provider- } \\
\text { directed decision } \\
\text { aid and } \\
\text { education vs. } \\
\text { education only }\end{array}$ & $\begin{array}{l}\text { Med: } 142 / \\
158(90 \%) \\
\text { filled Rx: } 94 / \\
109(86 \%) \\
\text { (if pharmacy } \\
\text { available) }\end{array}$ & $\begin{array}{l}\text { Med: } 110 / \\
139(79 \%) \\
\text { filled Rx: } \\
82 / 88(93 \%) \\
\text { (if pharmacy } \\
\text { available) }\end{array}$ & NR & $\begin{array}{l}>80 \% \text { of } \\
\text { days } \\
\text { covered } \\
(95 \text { vs. } \\
98 \%)\end{array}$ \\
\hline
\end{tabular}

*Unless otherwise specified, all levels were at the patient level

${ }^{\dagger}$ Treatment initiation was extrapolated from refusal of randomization after participated notified of the match vs. mismatch status

${ }^{*}$ Includes any mental health provider visit including primary care provider or visit for medication

${ }^{\S}$ Arean (2005) reports same intervention effects but by race: Psych visit: White 45\% (41-48\%) vs. 18\% (15-21\%); Black 41\% (30-51\%) vs. $12 \%$ (5-

19\%); Medication: White 65\% (62-68\%) vs. $18 \%$ (15-21\%); Black 55\% (40-58\%) vs. 49\% (40-58\%). Ayalon (2007) assesses intervention at one site with similar findings but more effective in Blacks than Whites

॥Results for medication only and therapy only are similar and significant

${ }^{" R e s u l t s ~ f o r ~ t h o s e ~ n o t ~ o n ~ a p p r o p r i a t e ~ t r e a t m e n t ~ a t ~ b a s e l i n e ~ a r e ~ n e a r l y ~ i d e n t i c a l ~ b u t ~ f r e q u e n c i e s ~ b y ~ i n t e r v e n t i o n ~ a r m ~ a r e ~ n o t ~ p r o v i d e d ~}$

\#Asked whether CBT used in last 2 months; also asked sources of help which included family friends, GP, and counselor/psychologist. For seeking help from counselor/psychologist: (CBT website vs. educational website vs control: 18 vs. 10 vs. $16 \%$; OR=1.93, 95\% CI 0.94-3.98; OR=1.01 95\% CI $0.58-1.76)$

Abbreviations: SCID DSMIV, Structured Clinical Interview for Diagnostic and Statistical Manual of Mental Disorders; CIDI, Composite International Diagnostic Interview; HRSD, Hamilton Rating Scale for Depression; CESD, Center for Epidemiologic Studies Depression Scale; QIDS, Quick Inventory of Depressive Symptomatology; GHQ, General Health Questionnaire; PHQ-D or 9, Patient Health Questionnaire; Psych, psychiatry or psychology, specifically entering or attending at least psychiatry-and psychology-related visit (including CBT and psychotherapy); Med, antidepressant medication; MI, motivational interviewing, motivation connotes motivational interviewing, empowerment, behavioral support, patient activation, and other motivational constructs (e.g., barriers assessment, concerns, outcome expectancy, goal setting); MH, mental health; Tx, treatment; N/A, not applicable; NR, not reported

coupled with a brief $(<15 \mathrm{~min})$ motivational phone call before appointments and after no shows significantly improved psychiatry attendance (vs. letter and reminder only) $(40 / 57(70 \%)$ vs. $18 / 56(32 \%))$ and total number of appointments. ${ }^{49}$
Motivation and Cultural Tailoring. Lara (2003) found no effect of a pre-post intervention of six 2-h group-based, culturally sensitive, educational sessions about depression and treatment options specific to women (vs. brief education session). ${ }^{46}$ 


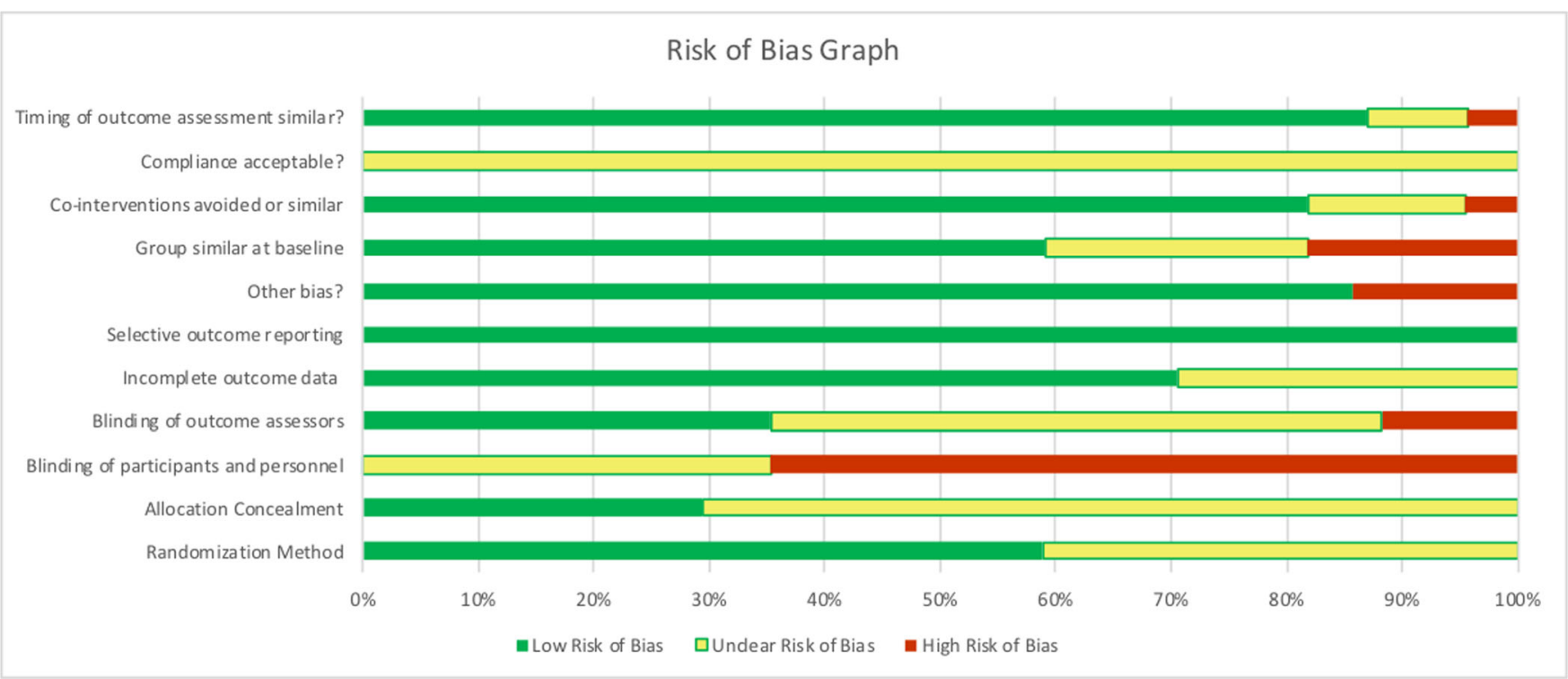

Figure 2 Risk of bias assessments.

Multilevel. Collaborative or Integrated Care. Among patients with substance abuse or depression, Bartels (2004) compared having onsite licensed mental health or substance abuse specialists who communicated with primary care providers and offered brief alcohol treatment options vs. transportation and cost assistance only (both with 2-4-week expedited appointments) and found significant improvement in mental health visit attendance (709/999 (71\%) vs. 499/1023 (49\%); OR = 2.57, 95\% CI 2.14 $3.08)^{38,40,41}$ but not depressive symptoms ${ }^{38}$ (Table 1, Online Supplemental 2). Wells (2000) and Jaycox (2003) found that a locally tailored quality improvement (QI) intervention that trained onsite nurses to educate and motivate providers and patients and provide medication management (QI Med) or counseling (QI Therapy) (vs. usual care) significantly improved receipt of any mental health treatment $(51 \%$ [48-54\%] vs. $40 \%$ [36-44\%]) and depressive symptoms ${ }^{43,47}$ as well as treatment completion rates. ${ }^{43}$ Arean $(2005,2007)$ found that onsite depression care managers who provided motivation, therapy, medication management, and treatment preference matching improved use of therapy, medications, and depressive symptoms across income and racial groups. ${ }^{37,39}$

Cultural Tailoring. Tailoring a community health clinic's collaborative care program to include Chinese-language PHQ-9s and culturally sensitive psychiatric assessments and referrals resulted in pre-post clinic level increase in treatment initiation from $19 / 296(7 \%)$ to $100 / 233(43 \%){ }^{48}$

Shared or Clinical Decision-making and Education. Le Blanc (2016) demonstrated that giving providers an antidepressant decision aid with an instructional session reduced patients' decisional conflict and improved satisfaction with treatment decisions, but not antidepressant initiation (142/ $158(90 \%)$ vs. $110.139(79 \%)$ ) or depressive symptoms. ${ }^{30}$

\section{Strength of Evidence}

We found moderate strength of evidence (SOE) for improving depression treatment initiation through collaborative/integrated care interventions (complex intervention) (3 of 3 studies reported statistically significant results for "any treatment initiation") (Table 2, Online Supplemental Table 3). Due to small sample sizes and risk of bias, we found low SOE for treatment preference matching (simple intervention) ( 2 of 2 studies beneficial). There was moderate SOE for the benefit of case management: Kim 2011 showed a trend toward benefit (OR = $1.51,95 \%$ CI 1.00-2.28) and Sirey (2016) (which added a treatment preference matching component) showed benefit (AOR $=2.40,95 \% \mathrm{CI} 1.17-4.93)$ but effect sizes and direction were consistent, suggesting overall benefit. For therapy outcomes only, there was insufficient evidence for motivation alone, cultural tailoring, and motivation with reminders (all had 1 beneficial, 1 null trial). There was insufficient (1 trial each) for case management, education, motivation with cultural tailoring, and shared decision-making across outcomes. In exploratory analyses, we found that the majority of interventions employed $\geq 1$ motivational strategy; 6 of 9 showed benefit (all of which were complex and individualized). Excluding four high-risk trials resulted in insufficient evidence for treatment preference matching and insufficient evidence for the effect of collaborative care studies on "any treatment" and medications. 
Table 2 Strength of Evidence for Engagement Strategies by Intervention Outcome

\begin{tabular}{|c|c|c|c|c|c|}
\hline \multirow[t]{2}{*}{$\overline{\text { Engagement strategy }}$} & \multicolumn{5}{|l|}{ Outcome* } \\
\hline & $\begin{array}{l}\text { Initiated any } \\
\text { depression } \\
\text { treatment }\end{array}$ & $\begin{array}{l}\text { Attended } \geq 1 \\
\text { mental health visit }\end{array}$ & $\begin{array}{l}\text { Initiated } \\
\text { antidepressant }\end{array}$ & $\begin{array}{l}\text { Persisted with } \\
\text { depression } \\
\text { treatment }\end{array}$ & $\begin{array}{l}\text { Depressive } \\
\text { symptoms }\end{array}$ \\
\hline $\begin{array}{l}\text { Case management* (appointment } \\
\text { facilitation and education and } \\
\text { motivation with or without reminders } \\
\text { or treatment preference matching) } \\
(n=2)\end{array}$ & $\begin{array}{l}\text { M (1 benefit, } 1 \text { no } \\
\text { benefit both with } \\
+ \text { effects })\end{array}$ & I (single RCT benefit) & $\begin{array}{l}\text { I (single RCT } \\
\text { benefit) }\end{array}$ & $\begin{array}{l}\text { I (single RCT } \\
\text { benefit) }\end{array}$ & $\begin{array}{l}\text { I (single RCT } \\
\text { no benefit) }\end{array}$ \\
\hline $\begin{array}{l}\text { Collaborative care (onsite access, } \\
\text { treatment preference matching with or } \\
\text { without motivation or education) } \\
(n=3)\end{array}$ & $\mathrm{M}(+)$ & $\mathrm{M}(+)$ & $\mathrm{M}(+)$ & $\mathrm{M}(+)$ & $\mathrm{M}(+)$ \\
\hline Cultural tailoring $(n=2)$ & & $\begin{array}{l}\text { I (smaller }[n=42] \\
\text { pre-post intervention } \\
\text { showed no } \\
\text { benefit; larger, pre-post } \\
\text { complex intervention } \\
\text { showed benefit) }\end{array}$ & & $\begin{array}{l}\text { I (single small } \\
\text { pre-post no benefit) }\end{array}$ & $\begin{array}{l}\text { I (single } \\
\text { pre-post no } \\
\text { benefit) }\end{array}$ \\
\hline Education $(n=1)$ & & $\begin{array}{l}\text { I (single RCT no } \\
\text { benefit) }\end{array}$ & $\begin{array}{l}\text { I (single RCT } \\
\text { no benefit) }\end{array}$ & & $\begin{array}{l}\text { I (single } \\
\text { RCT benefit) }\end{array}$ \\
\hline Motivation $(n=2)$ & & $\begin{array}{l}\text { I (complex website } \\
\text { benefit, simple leaflet } \\
\text { no benefit) }\end{array}$ & $\begin{array}{l}\text { I (single RCT } \\
\text { no benefit) }\end{array}$ & & $\begin{array}{l}\text { I (single } \\
\text { complex } \\
\text { RCT benefit) }\end{array}$ \\
\hline $\begin{array}{l}\text { Motivation and cultural tailoring } \\
(n=1)\end{array}$ & & $\begin{array}{l}\text { I (single pre-post no } \\
\text { benefit) }\end{array}$ & & & \\
\hline Motivation and reminder $(n=2)$ & & $\begin{array}{l}\text { I (motivational calls } \\
\text { benefit; leaflet no } \\
\text { benefit; both small } \\
\text { trials) }\end{array}$ & & $\begin{array}{l}\text { I (motivational calls } \\
\text { benefit; leaflet no } \\
\text { benefit) }\end{array}$ & \\
\hline $\begin{array}{l}\text { Shared/clinical decision-making and } \\
\text { education }(n=1)\end{array}$ & & & $\begin{array}{l}\text { I (single RCT } \\
\text { no benefit) }\end{array}$ & $\begin{array}{l}\text { I (single RCT no } \\
\text { benefit) }\end{array}$ & $\begin{array}{l}\text { I (single RCT } \\
\text { no benefit) }\end{array}$ \\
\hline Treatment preference matching $(n=2)$ & $\mathrm{L}(+)$ & & & $\begin{array}{l}\text { I ( } 1 \text { benefit, } 1 \text { no } \\
\text { benefit) }\end{array}$ & $\mathrm{L}(-)$ \\
\hline Complex interventions $(n=8)$ & $\mathrm{M}(+)$ & $\mathrm{M}(+)$ & $\begin{array}{l}\text { I ( } 3 \text { benefit, } 3 \\
\text { no benefit) }\end{array}$ & $\mathrm{M}(+)$ & $\mathrm{M}(+)$ \\
\hline Simple interventions $(n=6)$ & L (+) (preference) & $\mathrm{L}(-)$ & & $\mathrm{L}(-)$ & $\begin{array}{l}\text { I (single } \\
\text { pre-post trial } \\
\text { no benefit) }\end{array}$ \\
\hline
\end{tabular}

Definitions of grades of overall strength of evidence: High, high confidence that the evidence reflects the true effect. Further research is very unlikely to change our confidence in the estimate of effect; Moderate, moderate confidence that the evidence reflects the true effect. Further research may change our confidence in the estimate of the effect and may change the estimate; Low, low confidence that the evidence reflects the true effect. Further research is likely to change our confidence in the estimate of the effect and is likely to change the estimate; Insufficient, evidence either is unavailable or does not permit estimation of an effect. Most of which were due to the fact that only one study was included above; (+), benefit for outcome ( $p<0.05)$; and (-), null for outcome

https://effectivehealthcare.ahrq.gov/sites/default/files/pdf/methods-guidance-grading-evidence methods.pdf

\section{DISCUSSION}

We identified 16 (4 simple and 12 complex) interventions representing 8 strategies for increasing depression treatment initiation in primary care. A greater proportion of complex (8 of 12), including multilevel (4 of 5), interventions reported statistically significant effects on treatment initiation than simple patient-level ( 2 of 4 ) interventions. Further, we found low to moderate strength of evidence for benefit of complex interventions and for collaborative/ integrated care, case management, and treatment preference matching strategies. We found insufficient evidence for education, motivation (alone, with reminders or with cultural tailoring) and shared decision-making. Although our primary outcome of interest was treatment initiation, we also found moderate strength of evidence for complex, particularly collaborative care, interventions on treatment retention, and depressive symptom outcomes.
Barriers to initiating depression treatment when offered ${ }^{54}$ have been attributed to factors such as stigmatization, ${ }^{55,56} \mathrm{low}$ self-efficacy, ${ }^{57}$ and poor access to care. ${ }^{58}$ Treatment engagement research has sought to target these barriers, but often focuses on improving intermediate outcomes such as selfreported intention or provider behaviors (e.g., referral rates), ${ }^{59}$ and less often on actual depression treatment initiation. ${ }^{60}$ Identifying effective engagement strategies is essential to optimizing the effectiveness of depression treatment. ${ }^{61}$

Our study confirms that collaborative/integrated care, long shown to be effective in improving depression treatment and depressive symptoms in primary care settings, ${ }^{9,62-65,66,67,68}$ is an important complex, multilevel strategy for increasing treatment initiation. We also found some evidence that case management, another complex but patient-level strategy, may improve treatment initiation, similar to its effect on other health behaviors. ${ }^{34}$ Our review adds to the literature by highlighting 
promising active ingredients within these programs. All collaborative/integrative care interventions employed treatment preference matching or onsite access, with the later more effective than simply facilitating appointments, transportation and costs. ${ }^{38,40,41}$ Treatment preference matching, even alone, appeared to be an effective approach to increasing treatment initiation. Despite the importance of eliciting patient preference, ${ }^{69}$ providers often mismatch treatment (e.g., prescribing antidepressants in those preferring psychotherapy). ${ }^{70-74}$ Higher quality studies will be integral to establishing the benefit of patient preference matching alone and the degree to which it is a key ingredient of case management and ${\text { collaborative } \text { care }^{75}}^{7}$

We further highlight several strategies that warrant further study, such as cultural tailoring, motivation, and shared decision-making. Shared decision-making is emphasized in primary care, ${ }^{76-78}$ but we found no evidence that improving the process of decision-making only (including two studies not meeting inclusion criteria) changes behavior. ${ }^{30,79,80}$ Many posit shared decision-making improves provider guideline adherence $^{81}$ as well as affective-cognitive variables but not health behaviors in patients. ${ }^{82}$ It may be that treatment preference matching, a component of shared decision-making, is sufficient or an active ingredient. Some have suggested pairing decision aids with motivational interventions to alter patient behaviors. ${ }^{83}$ While we found insufficient evidence for motivation due to biased, small and inconsistent trials, most interventions employed $\geq 1$ motivational component $(n=9)$ and 6 of these showed benefit (all of which were complex and delivered actively, i.e., via calls or websites). Further highquality research is needed to understand whether motivational strategies are effective alone or need to be delivered within complex interventions. Technologies such as video-based multimedia $^{59}$ and patient portals may be useful for scaling motivational interventions. ${ }^{84}$

Relatedly, we demonstrate that complex interventions (e.g., more components, contacts, or duration) may be needed to improve treatment retention, depressive symptoms, and antidepressant treatment initiation. Because many simple interventions were not powered to assess depressive symptoms, further research is needed to elucidate differences between simple and complex interventions. One approach would be to implement simple interventions in integrated settings to improve initiation as well as foster retention and clinical outcomes.

There are several limitations to our review. We excluded non-English language studies, which may have contributed to publication bias. Relatedly, the majority of trials, including those with small samples, reported benefit. There was also wide heterogeneity in outcome measurement precluding metaanalysis, while the small number of trials within each strategy limited our ability pool effects in any single category. However, we applied a rigorous grading approach to determine SOE. Additionally, treatment initiation itself may be a low bar to achieve and insufficient for improving patient outcomes.
Nonetheless, treatment initiation remains a vital first step, and this remains one of the first systematic reviews to identify pragmatic interventions to improve depression treatment engagement behaviors in primary care settings. While we applied rigorous methods to categorize strategies, some there may have been overlap or mis-categorization. Finally, the included studies had moderate to high risk of bias and none were graded as high SOE, though this is common in behavioral interventions.

Overall, our review provides practical strategies for increasing depression treatment initiation in primary care. Patient engagement interventions that advocate feasibility with patients, providers, and organizational workflows will be key. Our review highlights the crucial need for more rigorous, low-risk studies confirming the effectiveness of these strategies, particularly collaborative care, case management, treatment matching, and motivation, which by their very nature make blinding difficult. Furthermore, research is needed understand how best to engage primary care team members in delivering these strategies. ${ }^{33}$

Corresponding Author: Nathalie Moise, MD, MS; Center for Behavioral Cardiovascular Health, Columbia University Medical Center, New York, NY, USA (e-mail: nm2562@cumc.columbia.edu).

Author Contributions Nathalie Moise, Louise Falzon, and Megan Obi had full access to all the data in the study and take responsibility for the integrity of the data and the accuracy of the data analysis. Study concept and design: Moise and Falzon; acquisition of data: Falzon; analysis and interpretation of data: Moise, Falzon, Obi, Kronish, Bryant, and Gonzalez; drafting of the manuscript: Moise, Falzon, Obi, and Kronish; critical revision of manuscript for important intellectual content: Moise, Falzon, Obi, Bryant, Gonzalez, Patel, and Kronish; statistical analysis: Moise, Falzon, and Obi; obtained funding: Moise and Kronish; and study supervision: Kronish.

Funding This research project is supported by funds from NHLBI (3 RO1 HL114924-03S1; HLO80477 and K24 HL111154) and AHRQ (R01 HS025198).

\section{Compliance with Ethical Standards:}

Conflict of Interest: The authors declare that they do not have a conflict of interest.

Transparency: Dr. Moise affirms that the manuscript is an honest, accurate, and transparent account of the study being reported; that no important aspects of the study have been omitted; and that any discrepancies from the study as planned (and, if relevant, registered) have been explained.

Prior Presentations: Parts of the results of this study were presented at the Academy Health Dissemination and Implementation Conference in December 2016.

\section{REFERENCES}

1. Williams JW Jr., Mulrow CD, Kroenke $\mathbf{K}$, et al. Case-finding for depression in primary care: a randomized trial. Am $\mathrm{J}$ Med 1999;106:36-43. 
2. Simon GE, VonKorff M. Recognition, management, and outcomes of depression in primary care. Arch Fam Med 1995;4:99-105.

3. Coyne JC, Fechner-Bates S, Schwenk TL. Prevalence, nature, and comorbidity of depressive disorders in primary care. Gen Hosp Psychiatry 1994; 16:267-76.

4. Bush DE, Ziegelstein RC, Tayback M, et al. Even minimal symptoms of depression increase mortality risk after acute myocardial infarction. Am J Cardiol 2001;88:337-41.

5. Gross R, Olfson M, Gameroff MJ, et al. Depression and glycemic control in Hispanic primary care patients with diabetes. J Gen Intern Med 2005;20:460-6.

6. Wulsin LR, Vaillant GE, Wells VE. A systematic review of the mortality of depression. Psychosom Med 1999;61:6-17.

7. Gan $\mathbf{Y}$, Gong $\mathbf{Y}$, Tong $\mathbf{X}$, et al. Depression and the risk of coronary heart disease: a meta-analysis of prospective cohort studies. BMC Psychiatry 2014; $14: 371$.

8. Unutzer J, Patrick DL, Diehr P, Simon G, Grembowski D, Katon W Quality adjusted life years in older adults with depressive symptoms and chronic medical disorders. Int Psychogeriatr 2000;12:15-33.

9. Unutzer $\mathbf{J}$ Harbin $\mathbf{H}$, Schoenbau M, Druss, B. The collaborative care model: an approach for integrated physical and mental health care in Medicaid health homes. Health Home Information Resource Center Brief May 2013. https://www.chcs.org/media/HH_IRC_Collaborative_Care_ Model_052113_2.pdf. Accessed 25 May 2018.

10. Williams DR, Gonzalez HM, Neighbors H, et al. Prevalence and distribution of major depressive disorder in African Americans, Caribbean blacks, and non-Hispanic whites: results from the National Survey of American Life. Arch Gen Psychiatry 2007;64:305-15.

11. Romera I, Montejo A, Aragones E, et al. Systematic depression screening in high-risk patients attending primary care: a pragmatic cluster-randomized trial. BMC Psychiatry 2013;13:83.

12. Smolderen KG, Buchanan DM, Amin AA, et al. Real-world lessons from the implementation of a depression screening protocol in acute myocardial infarction patients: implications for the American Heart Association depression screening advisory. Circ Cardiovasc Qual Outcomes 2011;4:283-92.

13. González HM, Vega WA, Williams DR, Tarraf W, West BT, Neighbors HW. Depression care in the united states: Too little for too few. Arch Gen Psychiatry 2010;67:37-46.

14. Thornicroft G, Chatterji S, Evans-Lacko S, et al. Undertreatment of people with major depressive disorder in 21 countries. Br J Psychiatry 2016;210: 119-24.

15. Olfson M, Blanco C, Marcus SC. TReatment of adult depression in the united states. JAMA Intern Med 2016;176:1482-91.

16. Richards DA, Borglin G. Implementation of psychological therapies for anxiety and depression in routine practice: two year prospective cohort study. J Affect Disord 2011;133:51-60.

17. Solberg LI, Crain AL, Jaeckels N, et al. The DIAMOND initiative: implementing collaborative care for depression in 75 primary care clinics. Implement Sci 2013;8:135.

18. Whitebird RR, Solberg LI, Jaeckels NA, et al. Effective Implementation of collaborative care for depression: what is needed? Am J Manag Care 2014;20:699-707

19. Sederer LI, Derman M, Carruthers J, Wall M. The New York State Collaborative Care Initiative: 2012-2014. Psychiatr Q 2016;87:1-23.

20. Regier DA, Goldberg ID, Taube CA. The de facto US mental health services system: a public health perspective. Arch Gen Psychiatry 1978;35(6):685-93.

21. Community and Mental Health Team. Psychological therapies: annual report on the use of IAPT services. England Health and Social Care Information Centre. Leeds U.K.: NHS Digital: 2015. Available at: https:// files.digital.nhs.uk/publication/q/1/psyc-ther-ann-rep-2016-17.pdf. Accessed 29 July 2018.

22. Aggarwal NK, Pieh MC, Dixon L, Guarnaccia P, Alegria M, LewisFernandez R. Clinician descriptions of communication strategies to improve treatment engagement by racial/ethnic minorities in mental health services: a systematic review. Patient Educ Couns 2016;99:198-209.

23. Gulliver A, Griffiths KM, Christensen H, Brewer JL. A systematic review of help-seeking interventions for depression, anxiety and general psychological distress. BMC Psychiatry 2012;12:81.

24. Interian A, Lewis-Fernandez $\mathbf{R}$, Dixon LB. Improving treatment engagement of underserved U.S. racial-ethnic groups: a review of recent interventions. Psychiatr Serv 2013;64:212-22.

25. Raue PJ, Schulberg HC, Heo M, Klimstra S, Bruce ML. Patients' depression treatment preferences and initiation, adherence, and outcome: a randomized primary care study. Psychiatr Serv 2009;60:337-43.
26. Craig P, Dieppe $\mathbf{P}$, Macintyre $\mathbf{S}$, Michie S, Nazareth I, Petticrew $\mathbf{M}$. Developing and evaluating complex interventions: the new Medical Research Council guidance. BMJ 2008;337:a1655.

27. Hawe P, Shiell A, Riley T. Complex interventions: how "out of control" can a randomised controlled trial be? Brit Med J 2004;328:1561-3.

28. Petticrew M. When are complex interventions 'complex"? When are simple interventions 'simple'? Eur J Public Health 2011;21:397-8.

29. Manhart LE, Holmes KK. Randomized controlled trials of individuallevel, population-level, and multilevel interventions for preventing sexually transmitted infections: what has worked? J Infect Dis 2005;191 Suppl 1:S7-24.

30. LeBlanc A, Herrin J, Williams MD, et al. Shared decision making for antidepressants in primary care: a cluster randomized trial. JAMA Intern Med 2015;175:1761-70.

31. Effective Public Health Practice Project (EPHPP). Quality assessment tool for quantitative studies. The Effective Public Health Practice Project (EPHPP), Hamilton, ON, Canada. 1998. http://www.nccmt.ca/knowledge-repositories/search/14. Accessed 25 May 2018.

32. Higgins JPT, Altman DG, Sterne JAC. Chapter 8: assessing risk of bias in included studies. In: Higgins JPT, Green S, editors. Cochrane handbook for systematic reviews of interventions: The Cochrane Collaboration; 2011.

33. Grande Sw, Faber MJ, Durand MA, Thompson R, Elwyn G. A classification model of patient engagement methods and assessment of their feasibility in real-world settings. Patient Educ Couns 2014;95:281-7.

34. Viswanathan M, Golin CE, Jones CD, et al. Interventions to improve adherence to self-administered medications for chronic diseases in the United States: a systematic review. Ann Intern Med 2012;157:785-95.

35. Berkman ND, Lohr KN, Ansari M, et al. Grading the strength of a body of evidence when assessing health care interventions for the effective health care program of the Agency for Healthcare Research and Quality: an update. Methods Guide for Comparative Effectiveness Reviews (Prepared by the RTI-UNC Evidence-based Practice Center under Contract No. 290-2007-10056-I). AHRQ Publication No. 13(14)-EHC130-EF. Rockville, MD: Agency for Healthcare Research and Quality. November 2013. Available at: https://effectivehealthcare.ahrq.gov/topics/methodsguidance-grading-evidence/methods. Accessed 25 May 2018

36. Alvidrez J, Snowden LR, Rao SM, Boccellari A. Psychoeducation to address stigma in black adults referred for mental health treatment: a randomized pilot study. Community Ment Health J 2009;45:127-36.

37. Arean PA, Ayalon $\mathbf{L}$, Hunkeler E, et al. Improving depression care for older, minority patients in primary care. Med Care 2005;43:381-90.

38. Arean PA, Ayalon L, Jin C, et al. Integrated specialty mental health care among older minorities improves access but not outcomes: results of the PRISMe study. Int J Geriatr Psychiatry 2008;23:1086-92.

39. Arean PA, Gum AM, Tang L, Unutzer J. Service use and outcomes among elderly persons with low incomes being treated for depression. Psychiatr Serv 2007;58:1057-64.

40. Ayalon L, Arean PA, Linkins $\mathbf{K}$, Lynch M, Estes CL. Integration of mental health services into primary care overcomes ethnic disparities in access to mental health services between black and white elderly. Am J Geriatr Psychiatry 2007;15:906-12.

41. Bartels SJ, Coakley EH, Zubritsky C, et al. Improving access to geriatric mental health services: a randomized trial comparing treatment engagement with integrated versus enhanced referral care for depression, anxiety, and at-risk alcohol use. Am J Psychiatry 2004;161:1455-62.

42. Christensen H, Leach LS, Barney L, Mackinnon AJ, Griffiths KM. The effect of web based depression interventions on self reported help seeking: randomised controlled trial [ISRCTN77824516]. BMC Psychiatry 2006;6:13.

43. Jaycox LH, Miranda J, Meredith LS, Duan N, Benjamin B, Wells K. Impact of a primary care quality improvement intervention on use of psychotherapy for depression. Ment Health Serv Res 2003;5: 109-20.

44. Kim SE, Le Blanc AJ, Michalopoulos C, et al. Does telephone care management help Medicaid beneficiaries with depression? Am J Manag Care 2011;17:e375-82.

45. Kwan BM, Dimidjian S, Rizvi SL. Treatment preference, engagement, and clinical improvement in pharmacotherapy versus psychotherapy for depression. Behav Res Ther 2010;48:799-804.

46. Lara MA, Navarro C, Rubi NA, Mondragon L. Two levels of intervention in low-income women with depressive symptoms: compliance and programme assessment. Int J Soc Psychiatry 2003;49:43-57.

47. Wells KB, Sherbourne C, Schoenbaum M, et al. Impact of disseminating quality improvement programs for depression in managed primary care: a randomized controlled trial. JAMA 2000;283:212-20. 
48. Yeung A, Shyu I, Fisher L, Wu S, Yang H, Fava M. Culturally sensitive collaborative treatment for depressed chinese americans in primary care. Am J Public Health 2010;100:2397-402.

49. Zanjani F, Miller B, Turiano N, Ross J, Oslin D. Effectiveness of telephone-based referral care management, a brief intervention to improve psychiatric treatment engagement. Psychiatr Serv 2008;59:776-81

50. Sirey JA, Banerjee S, Marino $\mathbf{P}$, et al. Improving mental health treatment initiation among depressed community dwelling older adults. Am J Geriatr Psychiatry 2016;24:310-9.

51. Delgadillo J, Morea O, Murphy E, Ali S, Swift JK. Can low-cost strategies improve attendance rates in brief psychological therapy? Double-blind randomized controlled trial. J Clin Psychol 2015;71:1139-52.

52. Kenwright M, Marks IM. Improving first attendance for cognitive behaviour therapy by a partial booking appointment method: two randomised controlled trials. J Ment Health 2003;12:385-92.

53. Larsen DL, Nguyen TD, Green RS, Attkisson CC. Enhancing the utilization of outpatient mental-health-services. Community Ment Health J 1983;19:305-20.

54. Tancredi DJ, Slee CK, Jerant A, et al. Targeted versus tailored multimedia patient engagement to enhance depression recognition and treatment in primary care: randomized controlled trial protocol for the AMEP2 study. BMC Health Serv Res 2013;13:141.

55. Graf J, Lauber C, Nordt C, Ruesch P, Meyer PC, Rossler W. Perceived stigmatization of mentally ill people and its consequences for the quality of life in a Swiss population. J Nerv Ment Dis 2004;192:542-7.

56. Givens JL, Katz IR, Bellamy S, Holmes WC. Stigma and the acceptability of depression treatments among African Americans and Whites. J Gen Intern Med 2007;22:1292-7.

57. Benassi VA, Sweeney PD, Dufour CL. Is there a relation between locus of control orientation and depression? J Abnorm Psychol 1988;97:357-67.

58. Brown A, Rice SM, Rickwood DJ, Parker AG. Systematic review of barriers and facilitators to accessing and engaging with mental health care among at-risk young people. Asia Pac Psychiatry 2016;8:3-22.

59. Kravitz RL, Franks P, Feldman MD, et al. Patient engagement programs for recognition and initial treatment of depression in primary care: a randomized trial. JAMA 2013;310:1818-28.

60. Mittler JN, Martsolf GR, Telenko SJ, Scanlon DP. Making sense of "consumer engagement" initiatives to improve health and health care: a conceptual framework to guide policy and practice. Milbank $\mathrm{Q}$ 2013;91:37-77.

61. Byatt N, Levin LL, Ziedonis D, Moore Simas TA, Allison J. Enhancing participation in depression care in outpatient perinatal care settings: a systematic review. Obstet Gynecol 2015;126:1048-58.

62. Thota AB, Sipe TA, Byard GJ, et al. Collaborative care to improve the management of depressive disorders: a community guide systematic review and meta-analysis. Am J Prev Med. 2012;42:525-38.

63. Siu AL, and the USPSTF. Screening for depression in adults: US Preventive Services Task Force Recommendation Statement. JAMA. 2016;315:380-7.

64. Rost K, Nutting P, Smith J, Werner J, Duan N. Improving depression outcomes in community primary care practice: a randomized trial of the quEST intervention. Quality enhancement by strategic teaming. J Gen Intern Med 2001;16:143-9.

65. Rost $\mathbf{K}$, Smith JL, Dickinson $\mathbf{M}$. The effect of improving primary care depression management on employee absenteeism and productivity. A randomized trial. Med Care 2004;42:1202-10.

66. Jarjoura D, Polen A, Baum E, Kropp D, Hetrick S, Rutecki G. Effectiveness of screening and treatment for depression in ambulatory indigent patients. J Gen Intern Med 2004;19:78-84.
67. Unützer J, Katon W, Callahan CM, et al. Collaborative care management of late-life depression in the primary care setting: a randomized controlled trial. JAMA 2002;288:2836-45.

68. Njeru JW, DeJesus RS, St Sauver J, et al. Utilization of a mental health collaborative care model among patients who require interpreter services. Int J Ment Health Syst 2016;10:15.

69. Lin P, Campbell DG, Chaney EF, et al. The influence of patient preference on depression treatment in primary care. Ann Behav Med 2005;30: 164-73.

70. Gum AM, Arean PA, Hunkeler E, et al. Depression treatment preferences in older primary care patients. Gerontologist 2006;46:14-22.

71. Chilvers $\mathbf{C}$, Dewey $\mathbf{M}$, Fielding $\mathbf{K}$, et al. Antidepressant drugs and generic counselling for treatment of major depression in primary care: randomised trial with patient preference arms. BMJ 2001;322:772-5.

72. Rost K, Humphrey J, Kelleher $\mathbf{K}$. Physician management preferences and barriers to care for rural patients with depression. Arch Fam Med 1994;3:409-14.

73. Davidson KW, Bigger J, Burg MM, et al. Centralized, stepped, patient preference-based treatment for patients with post-acute coronary syndrome depression: CODIACS vanguard randomized controlled trial. JAMA Intern Med. 2013;173:997-1004.

74. Davidson KW, Rieckmann N, Clemow L, et al. Enhanced depression care for patients with acute coronary syndrome and persistent depressive symptoms: coronary psychosocial evaluation studies randomized controlled trial. Arch Intern Med 2010;170:600-8.

75. Gelhorn HL, Sexton CC, Classi PM. Patient preferences for treatment of major depressive disorder and the impact on health outcomes: a systematic review. Prim Care Companion to CNS Disord 2011;13:PCC.11r01161.

76. Stewart MA. Effective physician-patient communication and health outcomes: a review. CMAJ 1995;152:1423-33.

77. Institute of Medicine. Crossing the Quality Chasm: A New Health System for the 21st Century. Washington, DC: The National Academies Press: 2001

78. Weobong B, Weiss HA, McDaid D, et al. Sustained effectiveness and cost-effectiveness of the Healthy Activity Programme, a brief psychological treatment for depression delivered by lay counsellors in primary care: 12 month follow-up of a randomised controlled trial. PLoS Med 2017;14:e1002385.

79. Alegria M, Carson N, Flores $\mathbf{M}$, et al. Activation, self-management, engagement, and retention in behavioral health care: a randomized clinical trial of the DECIDE intervention. JAMA Psychiatry 2014;71:557-65.

80. Loh A, Simon D, Wills CE, Kriston L Niebling W, Harter M. The effects of a shared decision-making intervention in primary care of depression: a cluster-randomized controlled trial. Patient Educ Couns 2007:67:32432.

81. Clever SL, Ford DE, Rubenstein LV, et al. Primary care patients' involvement in decision-making is associated with improvement in depression. Med Care 2006;44:398-405.

82. Shay LA, Lafata JE. Where is the evidence? A systematic review of shared decision making and patient outcomes. Med Decis Making 2015;35:114-31.

83. Alegria M, Polo A, Gao S, et al. Evaluation of a patient activation and empowerment intervention in mental health care. Med Care 2008;46:247-56.

84. Volpp KG, Mohta NS. Patient engagement survey: how to hardwire engagement into care delivery processes. Waltham, MA: NEJM Group: 2017. 